\title{
Proporção e tendência temporal das cesáreas nas regionais de saúde do Estado do Paraná, 2003 a 2014
}

\section{Proportion and time trend of cesarean sections in the health region in Paraná State, from 2003 to 2014}

\author{
Valéria Christino da Silva Amari (D), Ana Claudia Garabeli Cavalli Kluthcovsky² (D), \\ Marcos Nader Amari' (D), Erildo Vicente Müller³ (D) \\ 'Hospital Universitário Regional dos Campos Gerais - Ponta Grossa (PR), Brasil. \\ ${ }^{2}$ Departamento de Medicina, Universidade Estadual de Ponta Grossa (UEPG) - Ponta Grossa (PR), Brasil. \\ ${ }^{3}$ Departamento de Enfermagem e Saúde Pública, Universidade Estadual de Ponta Grossa (UEPG) - Ponta Grossa (PR), Brasil.
}

Como citar: Amari, V. C. S., Kluthcovsky, A. C. G. C., Amari, M. N., \& Müller, E. V. Proporção e tendência temporal das cesáreas nas regionais de saúde do Estado do Paraná, 2003 a 2014. Cad Saúde Colet, 2021;29(1):25-35. https://doi.org/10.1590/1414-462X202129010440

\section{Resumo}

Introdução: As taxas de cesáreas vêm aumentando no Brasil e no mundo, com maior preocupação em relação às consequências para a saúde materno-infantil. Objetivo: Analisar as proporções e a tendência temporal das cesáreas nas 22 Regionais de Saúde do Estado do Paraná. Método: Estudo ecológico de série temporal, com dados coletados do Sistema de Informações sobre Nascidos Vivos. As análises das proporções e da tendência temporal das cesáreas foram realizadas entre 2003 e 2014, por triênios. A tendência temporal foi calculada utilizando-se modelos de regressão linear simples, com nível de significância de 5\%. Resultados: As maiores proporções de cesáreas ocorreram no triênio entre 2012 e 2014 (53,8\% a 80,2\%), exceto para a 1ª Regional de Saúde (Paranaguá), com maiores proporções entre 2009 e 2011. Todas as regionais apresentaram tendência crescente na proporção de cesáreas (todos valores de $\mathrm{p}<0,05)$, exceto a $1^{\text {a }}$ Regional de Saúde que permaneceu com tendência estacionária $(p=$ $0,10)$. A grande maioria das Regionais de Saúde do Paraná apresentou proporção de cesáreas superior à do Brasil, para todos os triênios analisados. Conclusão: Os resultados apontam elevadas proporções de cesáreas nas Regionais de Saúde do Estado do Paraná e a grande maioria das regionais apresentou tendência crescente de cesáreas. Palavras-chave: cesárea; parto; saúde materno-infantil; estudos de séries temporais; epidemiologia.

\begin{abstract}
Background: Caesarean rates are increasing in Brazil and worldwide, and there is a concern regarding the consequences of this increase for maternal and child health. Objective: This study aimed to analyse the proportions and the time trend of cesarean sections in the Health Region of Paraná State. Method: This is a time-series ecological study using data from the Information System on Live Births. The analyzes of the proportions and trends of cesarean sections were performed between 2003 and 2014, in three-year periods. Linear regression was used to analyze cesarean rate trends, with the significance level set at $5 \%$. Results: The largest proportions of cesarean sections occurred between 2012 and 2014 (53.8\% to 80.2\%), except for the $1^{\text {st }}$ Health Region (Paranaguá), with larger proportions between 2009 and 2011. All the regional ones presented an increasing trend in the proportion of cesarean sections (all $p$ values $<0.05$ ), except the $1^{\text {st }}$ Health Region that remained with a steady trend $(p=0.10)$. The vast majority of the Health Region of Paraná State presented a higher proportion of cesarean sections than Brazil, for all three-year
\end{abstract}

Este é um artigo publicado em acesso aberto (Open Access) sob a licença Creative Commons Attribution, que permite uso, distribuição e reprodução em qualquer meio, sem restrições desde que o trabalho original seja corretamente citado.
Trabalho realizado na Universidade Estadual de Ponta Grossa (UEPG) - Ponta Grossa (PR), Brasil.

Correspondência: Valéria Christino da Silva Amari. E-mail: vcsamari@gmail.com

Fonte de financiamento: nenhuma.

Conflito de interesses: nada a declarar.

Recebido em: Set. 03, 2018. Aprovado em: Fev. 28, 2020 
periods. Conclusion: The results indicate high proportions of cesarean sections in the Health Region in Paraná State and the vast majority showed an increasing trend of cesarean sections.

Keywords: cesarean section; parturition; maternal and child health; time-series studies; epidemiology.

\section{INTRODUÇÃOO}

Há muito tempo a Organização Mundial da Saúde (OMS) preconiza que o objetivo da assistência ao parto seja o acompanhamento da mulher e do concepto, com o mínimo de interferência médica, buscando garantir a segurança de ambos'. Contudo, nos últimos anos as taxas de cesáreas tiveram um aumento muito importante, tanto que atualmente uma em cada cinco mulheres no mundo dá à luz por essa via de parto².

O aumento na proporção de cesáreas é fato que pode ser constatado ao longo dos anos na prática e também comprovado por relatos da literatura ${ }^{3,4}$. O Brasil ocupa lugar de destaque entre os países que mais realizam cesáreas no mundo, junto com a China ${ }^{5,6}$. Esses dois países juntos são responsáveis por metade de todas as cesáreas realizadas no mundo 5 . Além da China, outros países que apresentam altas taxas são a Turquia, o México, a Itália e os Estados Unidos ${ }^{7}$.

O crescimento das taxas de cesáreas é bastante preocupante. Uma pesquisa multicêntrica realizada entre 2004 e 2005 na África e nas Américas e entre 2007 e 2008 na Ásia, em 24 países e em 373 estabelecimentos de saúde, buscou investigar a relação entre a cesárea sem indicação médica e os resultados maternos graves. De um total de 286.565 partos analisados, a taxa geral de cesáreas foi de $25,7 \%$. As cesáreas foram associadas a um risco intrínseco de aumento de resultados maternos graves e, portanto, devem ser realizadas quando um benefício claro é antecipado, que poderia compensar os custos mais altos e os riscos adicionais associados a esta operação ${ }^{8}$. Outros estudos demonstraram que o excesso de cesáreas pode resultar em maior morbidade para a mãe ${ }^{9}$ e para o recém-nascido ${ }^{10}$.

Além disso, o parto cesáreo representa um gasto $32 \%$ maior do que o parto normal, caso não ocorram complicações, segundo estudo que comparou os custos do parto vaginal e da cesariana eletiva sem indicação clínica, para gestantes de risco habitual em três maternidades públicas situadas na região Sudeste ${ }^{11}$. Sendo assim, o aumento do número de cesáreas consideradas desnecessárias ou sem indicação obstétrica acaba gerando gastos excessivos ${ }^{12}$.

Diante desse contexto, os resultados obtidos em estudos sobre o aumento das proporções e tendências das cesáreas podem servir como base de argumentos, justificativas ou mesmo como princípio para avaliações ou tomadas de decisões, em todos os setores. Além disso, podem direcionar maior cautela na indicação do tipo de parto, coibindo excessos e melhorando a qualidade da assistência ao parto, evitando iatrogenias e facilitando o acesso a todas as pacientes que realmente necessitem deste benefício, com segurança e respeito ao nascer.

Este estudo objetivou analisar as proporções e a tendência temporal das cesáreas nas Regionais de Saúde do Estado do Paraná, entre 2003 e 2014.

\section{MÉTODO}

O presente estudo é do tipo ecológico, de série temporal. Os dados foram coletados do Sistema de Informações sobre Nascidos Vivos (Sinasc) ${ }^{13}$, do Departamento de Informática do Sistema Único de Saúde (Datasus) para cada ano, por Regional de Saúde do Estado do Paraná, segundo nascidos vivos por residência da mãe e tipo de parto (vaginal, cesárea e ignorado).

Para o cálculo das proporções das cesáreas foram selecionados os números de partos vaginais e partos cesáreos de nascidos vivos de mães residentes na Regional de Saúde (RS). Os partos foram corrigidos para subregistros, onde os partos ignorados foram distribuídos entre partos vaginais e cesáreas de acordo com a porcentagem encontrada em cada grupo. Para cada Regional de Saúde foi calculada a proporção de cesáreas utilizando a equação: número de nascidos vivos por cesárea na Regional de Saúde no triênio dividido pelo número total de nascidos vivos na Regional de Saúde no triênio, multiplicado por 100.

A tendência temporal das proporções de cesáreas de nascidos vivos nas Regionais de Saúde do Estado do Paraná foi analisada de 2003 a 2014, agrupadas em por quatro triênios, a saber de 2003 a 2005, 2006 a 2008, 2009 a 2011 e 2012 a 2014. Também foi realizado o cálculo 
da variação percentual (VP) das proporções de cesáreas ao longo do período analisado, por Regional de Saúde. Para cada Regional de Saúde foi calculada a variação percentual das cesáreas utilizando a equação: número de nascidos vivos por cesárea na Regional de Saúde de 2012 a 2014 dividido pelo número de nascidos vivos por cesárea na Regional de Saúde de 2003 a 2005, menos 1 (um), multiplicado por 100.

A análise da tendência temporal foi feita utilizando-se modelos de regressão ${ }^{14}$. A série foi modelada após o processo de suavização com regressão polinomial. O processo de modelagem teve início com o modelo de regressão linear simples, onde a variável dependente (y) correspondeu às proporções de cesáreas e à variável independente $(x)$ aos triênios pesquisados. $\mathrm{O}$ modelo linear foi definido como $\mathrm{y}=\mathrm{b} 0+\mathrm{b} 1 \mathrm{x}$, onde b0 corresponde ao coeficiente médio para o período e b1 para o incremento (aumento ou diminuição) no período. Em seguida foram testados os modelos de segundo e terceiro graus. Considerou-se como melhor modelo o que apresentou maior significância estatística, além de resíduos sem vícios e o de menor ordem, quando ocorreu semelhança entre dois modelos do ponto de vista estatístico. Como medida de precisão foi utilizado o coeficiente de determinação $\left(R^{2}\right)$. Foram realizadas avaliações de normalidade das variáveis utilizando-se histogramas e teste de Shapiro-Wilk. Para fins de comparação, os mesmos cálculos foram feitos para o Estado do Paraná e para o Brasil, utilizando-se dados do Sinasc.

Os dados foram processados no banco de dados Excel 2010 e para os cálculos utilizou-se o programa estatístico Statistical Package for Social Science (IBM SSPS Statistics), versão 15.0. 0 nível de significância adotado foi de $5 \%$.

Obedecendo a Resolução n 466/2012 do Conselho Nacional de Saúde, que dispõe sobre as Diretrizes e Normas Regulamentadoras de Pesquisa envolvendo Seres Humanos, o projeto foi submetido e aprovado pela Comissão de Ética em Pesquisa (Coep) da Universidade Estadual de Ponta Grossa, sob protocolo n 1.652.527, CAAE n 57949216.9.0000.0105.

\section{RESULTADOS}

No Estado do Paraná, no período de 2003 a 2014, ocorreu um total de 1.853 .325 partos de nascidos vivos, incluindo partos vaginais, cesáreos e ignorados. Do total, após correção proporcional dos partos ignorados ( $0,04 \%)$, foram realizados $44,1 \%$ partos vaginais e $55,9 \%$ partos cesáreos ao longo do período.

As proporções de cesáreas em relação ao total de partos e a tendência temporal, para cada Regional de Saúde do Paraná, para o Estado do Paraná e para o Brasil, por triênios, podem ser observadas na Tabela 1.

As maiores proporções de cesáreas foram observadas no triênio 2012 a 2014, exceto para a $1^{\text {a }}$ Regional de Saúde (Paranaguá) que apresentou menor proporção de cesáreas no triênio 2012 a 2014 em relação ao triênio 2009 a 2011.

Considerando as proporções de cesáreas de todos os triênios, a menor proporção foi a da Regional de Telêmaco Borba (21 $1^{\mathrm{a}}$ ) com 31,6\% no triênio 2003 a 2005 e a maior proporção foi a da Regional de Paranavaí (14ª) com 80,2\% no triênio 2012 a 2014. Além disso, no triênio 2012 a 2014, todas as proporções de cesáreas ultrapassaram os 50\%. Comparando-se com as proporções de cesáreas do Brasil, no triênio 2012 a 2014, 19 (86,4\%) Regionais de Saúde apresentaram proporções de cesáreas maiores que a média do país para o mesmo triênio (56,5\%).

As Regionais de Saúde com as menores proporções de cesárea para o triênio 2012 a 2014 foram a $5^{a}$ (Guarapuava) com 53,8\%, a 1a (Paranaguá) com 53,9\% e a 3a (Ponta Grossa) com $55,8 \%$. Todas essas regionais pertencem à Macrorregional Leste. A maior proporção de cesáreas para o triênio 2012 a 2014 foi a da 14 ${ }^{\mathrm{a}}$ Regional (Paranavaí), seguida pela 12a (Umuarama) e 15a (Maringá) com 80,2\%, 77,5\% e 76,7\%, respectivamente, todas pertencentes à Macrorregional Noroeste.

Comparadas com o Paraná, 10 regionais $(45,5 \%)$ apresentaram maiores proporções de cesáreas no triênio 2003 a 2005, 12 (54,5\%) no triênio 2006 a 2008, 9 (41,0\%) no triênio 2009 a 2011 e 11 (50,0\%) no triênio 2012 a 2014 (Tabela 1). 


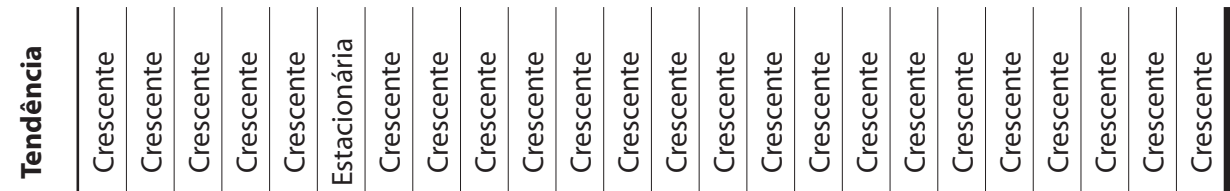

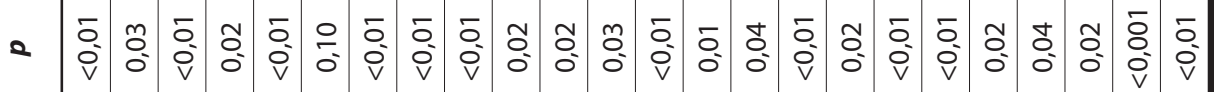

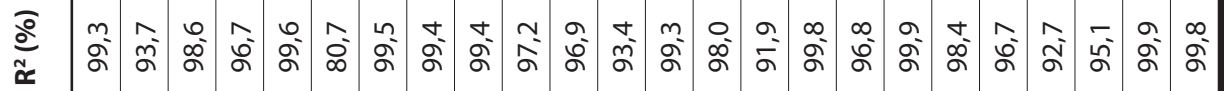

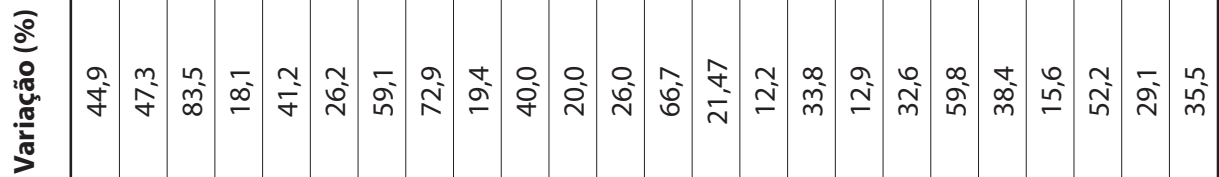

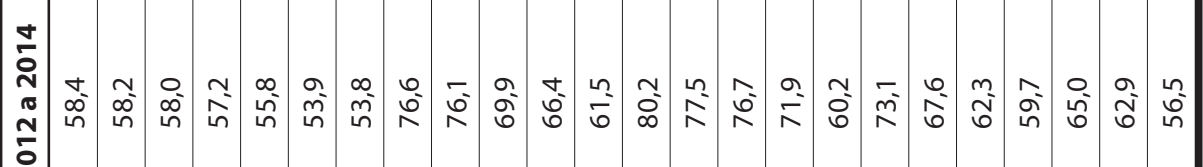

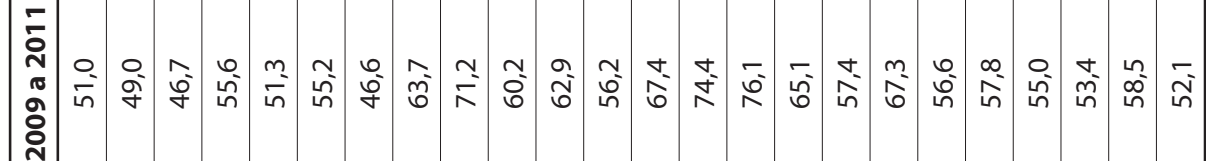

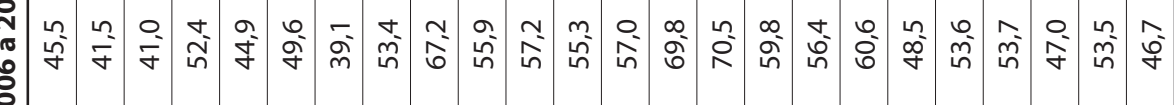

น

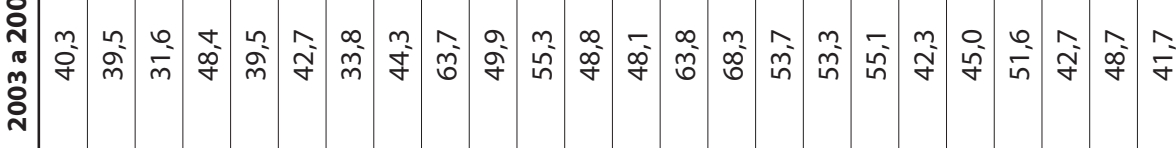


Comparadas com o Brasil, 18 regionais $(81,8 \%)$ apresentaram maiores proporções de cesáreas no triênio 2003 a 2005, 17 (77,2\%) no triênio 2006 a 2008, 17 (77,2\%) no triênio 2009 a 2011 e 19 (86,3\%) no último triênio. Importante ressaltar que as regionais $11^{\mathrm{a}}$ (Campo Mourão), 12a (Umuarama), 15a (Maringá), 16 (Apucarana), 17a (Londrina), 18a (Cornélio Procópio) e 20a (Toledo) apresentaram proporções de cesáreas maiores que o Paraná, para todos os quatro triênios analisados. Todas essas regionais pertencem às Macrorregionais Norte e Noroeste, exceto a $20^{\mathrm{a}}$ que pertence à Macrorregional Oeste (Tabela 1).

Quanto à variação percentual da proporção de cesáreas no período analisado, a $21^{\text {a }}$ Regional de Saúde (Telêmaco Borba) foi a que apresentou a maior variação percentual, de $83,5 \%$, seguida da 22a Regional de Saúde (Ivaiporã) com 72,9\% e da 14a (Paranavaí) com $66,7 \%$. As menores variações percentuais foram para a $15^{a}$ (Maringá) com $12,2 \%$, seguida da $13^{\mathrm{a}}$ (Cianorte) com 12,9\% e 9a (Foz do Iguaçu) com 15,6\%. O Estado do Paraná apresentou uma variação percentual de $29,1 \%$ e o Brasil de $35,5 \%$.

Na análise de tendência das proporções de cesáreas observou-se que houve um padrão crescente nas curvas de estimação para todas as Regionais de Saúde, exceto para a $1^{a}$ Regional de Saúde (Paranaguá), única que mostrou tendência estacionária $(p=0,10)$. O Estado do Paraná e Brasil também apresentaram tendência crescente nas proporções de cesáreas.

A Figura 1 apresenta as proporções de cesáreas em relação ao total de partos e a tendência temporal para cada Macrorregional de Saúde do Paraná e para o Estado do Paraná, por triênios.

Observa-se que a Macrorregional Noroeste apresentou as maiores proporções de cesáreas em todos os triênios analisados. No ultimo triênio (2012 a 2014), apenas a Macrorregional de Saúde Leste apresentou proporção de cesáreas menor que o Estado do Paraná.

A Tabela 2 apresenta o total de partos e de cesáreas nas Regionais de Saúde do Paraná e percentuais em relação aos totais do estado, por triênios, no período analisado. Observa-se que, em relação ao total de cesáreas no Estado do Paraná, por triênios, as regionais que mais contribuíram para o total do estado foram, em ordem decrescente, a $2^{\text {a }}$ Regional de Saúde com sede em Curitiba (proporções variando de $29,8 \%$ a 31,3\%), a $17^{\text {a }}$ com sede em Londrina (proporções variando de $8,0 \%$ a 8,5\%) e a $15^{\text {a }}$ com sede em Maringá (proporções variando de $7,8 \%$ a $8,1 \%)$. As demais regionais apresentaram proporções bem menores, variando de 1,2\% a $5,4 \%$.

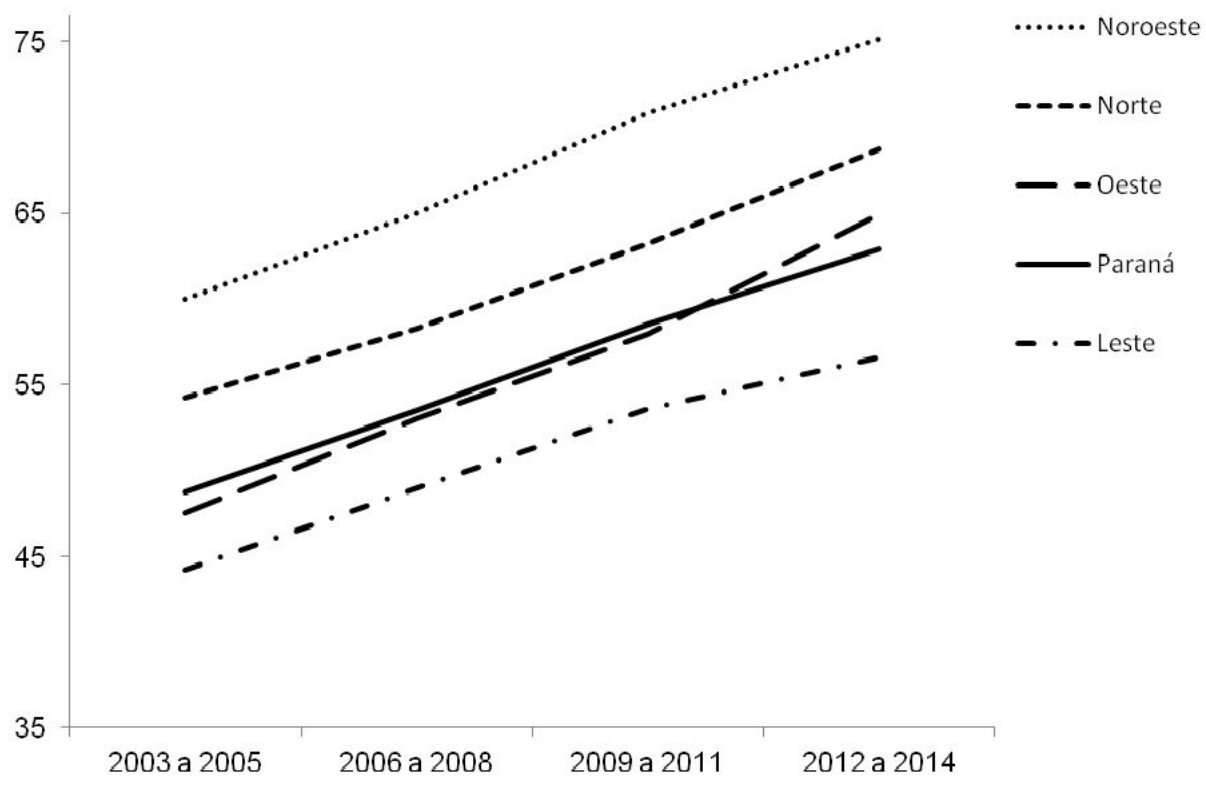

Figura 1. Proporção de partos cesáreos, por triênios, Macrorregionais de Saúde do Paraná e Estado do Paraná, 2003 a 2014 


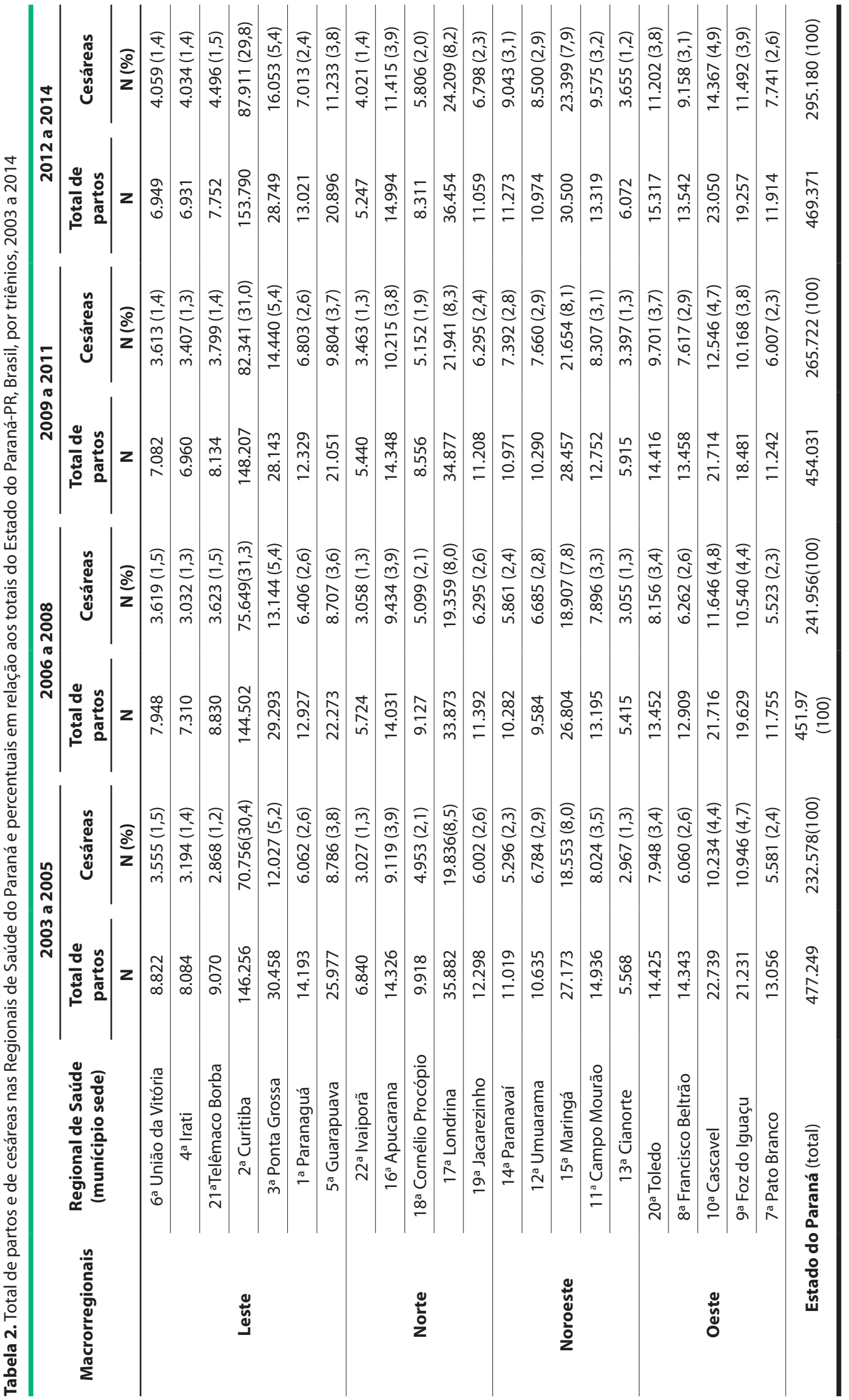




\section{DISCUSSÃO}

Em 1985 a OMS publicou a primeira referência para taxas de cesáreas, preconizando taxas entre $10 \%$ e $15 \%$ em relação ao total de partos ${ }^{1}$. Mais recentemente, a mesma organização desenvolveu uma ferramenta avaliativa levando em conta as características de cada gestante e de suas gestações que poderiam influenciar nas taxas de cesáreas de cada região, considerando 43 países, dentre eles o Brasil, desenvolvida e validada com mais de 10 milhões de nascimentos ${ }^{15}$. Para a população brasileira, devido às suas características específicas, foi recomendada uma proporção de cesáreas em torno de $25 \%$ a $30 \%{ }^{16}$. Mesmo considerando as recomendações mais recentes da OMS, as proporções de cesáreas para todas as Regionais de Saúde do Paraná, em todos os triênios analisados, foram superiores a $30 \%$.

Vale ressaltar que uma das regionais em estudo chegou a $80,2 \%$ de cesáreas no último triênio (2012 a 2014). Além disso, no último triênio analisado todas as proporções de cesáreas ultrapassaram os $50 \%$, sendo que $86,4 \%$ das regionais apresentaram proporções maiores que a média do país para o mesmo período.

Esses resultados são muito preocupantes, pois estudos evidenciaram que taxas de cesariana maiores que cerca de $10 \%$ ao nível da população não estão associadas à diminuição das taxas de mortalidade materna e neonatal ${ }^{17}$. Esses fatos podem indicar que a realização desmensurada das cesáreas não traz o benefício esperado ${ }^{18}$, além de poder aumentar os desfechos adversos tanto para a mãe ${ }^{8,9,19}$ quanto para o recém-nascido ${ }^{10}$.

O padrão da proporção de cesáreas encontrado acima do recomendado pela OMS também foi observado em outras áreas do Brasil, porém com algumas diferenças regionais. Taxas de cesáreas em torno de 35\% a 45\% foram observadas especialmente nas regiões Norte e Nordeste, enquanto nos estados das regiões Sul, Sudeste e Centro-Oeste as taxas foram de $55 \%$ a $65 \%{ }^{20}$.

Em outros países, como na China, foi descrita uma proporção de cesáreas de 54,9\% em $2011^{21}$, semelhante à de algumas Regionais de Saúde deste estudo, nos três primeiros triênios avaliados.

Por outro lado, taxas menores de cesáreas quando comparadas aos resultados deste estudo foram descritas em alguns países: de 30,5\% nos Estados Unidos entre 2002 e $2008^{22}$, $28,6 \%$ no Peru em $2014^{23}$. Taxas bem menores foram observadas em 2012: de 14,7\% na Finlândia, de $13,9 \%$ na Bósnia-Herzegovina e 1,7\% no Timor- Leste ${ }^{17}$.

Importante ressaltar que taxas muito baixas de cesáreas parecem ser insuficientes para uma atenção adequada. Em estudo ecológico realizado entre 1991 e 2003, utilizando dados de 119 países de baixa, média e alta renda, nenhuma associação foi observada em países de média renda (36\% com taxas de cesáreas acima de $20 \%$ ) e alta renda (36\% com taxas de cesáreas acima de $20 \%$ ) entre taxas de cesáreas e mortalidade neonatal ou mortalidade materna. Em países de baixa renda (76\% com taxas de cesáreas entre 0 e $10 \%)$, uma correlação linear negativa e estatisticamente significativa foi observada entre as taxas de cesárea e mortalidade neonatal e materna ${ }^{24}$.

De modo geral, as taxas de cesáreas apresentam-se mais elevadas em países desenvolvidos e na América Latina, como vistos no Brasil (56,6\%) e no México (49\%), em 2013. Contudo, países mais desenvolvidos, como a Holanda e Finlândia, por exemplo, apresentaram taxas baixas de cesáreas, de 15,6\% e 15,8\%, respectivamente, para o mesmo ano ${ }^{25}$. Logo, as taxas de cesáreas variam entre países, sendo fruto de diversos fatores envolvidos, como, por exemplo, diferenças socioeconômicas e relacionadas à imigração. Em um estudo ecológico, a mediana das taxas de cesárea foi menor nos países de baixa renda do que nos de média e alta renda ${ }^{24}$. Pesquisa realizada no Canadá com dados de 3.500 mulheres com gestação de baixo risco, as taxas de cesárea diferiram pelo status de migração. As mulheres refugiadas que migraram para o Canadá do Sudeste e Ásia Central experimentaram excesso de cesáreas, enquanto os refugiados da América Latina experimentaram menos, comparados aos nascidos no Canadáz ${ }^{6}$.

Dentre as causas da prática excessiva da cesárea no Brasil pode-se citar o modo como a assistência ao nascimento é organizada em nosso país, bastante centrado na ação individual dos profissionais em detrimento da abordagem em equipe multidisciplinar; a qualidade dos serviços de saúde de assistência ao parto; a assistência pré-natal e as características socioculturais ${ }^{16}$. 
Ao se analisar a tendência temporal da proporção das cesáreas no período de 2003 a 2014, observou-se um padrão crescente para todas as Regionais de Saúde, exceto para a $1^{\text {a }}$ Regional de Saúde (Paranaguá), que mostrou tendência estacionária. O mesmo padrão crescente se observou para o Estado do Paraná e o Brasil. O estado apresentou no primeiro triênio do estudo $48,7 \%$ de cesáreas, passando de $50 \%$ no segundo triênio, antes do país, que só ultrapassou $50 \%$ no terceiro triênio, com 52,1\%.

Vários estudos nacionais ${ }^{27-30} \mathrm{e}$ internacionais $\mathrm{s}^{2,6,17,31-34}$ demonstraram a tendência crescente do parto cesáreo, como observado nas Regionais de Saúde do Estado do Paraná.

No Estado do Piauí, a proporção de partos cesáreos em primíparas registrados no Sistema de Informações sobre Nascidos Vivos (partos hospitalares) passou de 34,4\% em 2000 para 51,1\% em $2011^{28}$. Outro estudo encontrou um aumento importante nas taxas de cesáreas nas regiões do Brasil: de $20,5 \%$ na região Nordeste, $18,0 \%$ na região Sul, $15,4 \%$ na região Centro-Oeste, $14,8 \%$ na região Norte e de $12,9 \%$ na região Sudeste ${ }^{29}$. Estudos de séries temporais realizados sobre os partos no Brasil encontrou um crescimento significativo dos partos cesáreos. $O$ crescimento da taxa de cesáreas no país entre 1999 e 2013 foi de 63,68\%, em conjunto com o aumento das consultas de pré-natal e hospitalização dos partos, com significativa redução dos partos vaginais ${ }^{30}$.

Esse padrão de aumento de cesáreas também pode ser observado em outros países. Por exemplo, a China teve um dos maiores aumentos de taxa de cesáreas no mundo, de no máximo 10\% em meados de 1980 para praticamente um total de 50\% de nascimentos por cesárea em $2010^{31}$. Em pesquisa realizada em cinco hospitais públicos e três privados em Puebla, México, também foi observado aumento da prevalência de cesáreas. Em 2012 eram 45,2\% dos partos, passando para 57,3\% em 2014². Em análise das tendências de cesáreas em 121 países de 1990 a 2014, a taxa média global de cesáreas apresentou um aumento de 12,4\% (de 6,7\% para $19,1 \%)$, com taxa média anual de aumento de $4,4 \%$. Os maiores aumentos absolutos ocorreram na América Latina e no Caribe (de 19,4\%, de 22,8\% para 42,2\%) 2 . Outro estudo realizado em regiões do Iraque demonstrou aumento da taxa de cesáreas no Iraque de 18\% em 2008 para $24,4 \%$ em $2012^{33}$.

Embora tenha existido uma variação na intensidade da tendência do crescimento das taxas de cesáreas em termos mundiais, com algumas regiões demostrando crescimento mais acentuado do que outras, a realização da cesárea em todo o mundo continua a despertar preocupação. Além do aumento dessa cirurgia, também há falta de consenso sobre a taxa apropriada de riscos e os custos de curto e longo prazo ${ }^{2}$.

Estudos vêm demonstrando que existe maior morbidade para a gestante após a realização da cesárea ${ }^{19}$. Há uma grande variedade e complexidade nos motivos para a escolha do tipo de parto, que, em conjunto ou isoladas, impulsionam o aumento das taxas de cesáreas. Diante disso, é primordial a avaliação e divulgação da informação científica em relação a algumas práticas comuns na atenção à operação cesariana programada, para fornecer subsídios e orientação no sentido de promover e proteger a saúde e o bem-estar da gestante e da criança ${ }^{16}$.

Nesse sentido, devem-se considerar as grandes mudanças que ocorrem na sociedade, no status da mulher e na medicina. Em adição, as diferenças entre os países com relação à realidade socioeconômica, cultural e etnográfica sugerem que uma taxa global de cesáreas fixa não deveria existir. Ao contrário, a taxa de cesárea poderia ser ajustada para cada país, levando em consideração as suas necessidades específicas ${ }^{25}$.

Diante do exposto, muitas ações foram colocadas em prática para valorização e segurança do parto normal, como uma tentativa de diminuir as cesáreas desnecessárias. Em nível mundial, a OMS concluiu que a Classificação de Robson é o sistema mais adequado para preencher as necessidades locais e internacionai ${ }^{34}$. Mais recentemente, em 2017, o Guia de Implementação da Lista de Verificação da OMS para Partos Seguros foi outro instrumento criado com o intuito de trazer mais segurança ao parto ${ }^{35}$.

A nível nacional, foi criado o projeto Parto Adequado $^{36}$ e, em 2016, o Ministério da Saúde (MS) criou os Protocolos Clínicos e Diretrizes Terapêuticas de Atenção à Gestante: a operação cesariana ${ }^{16}$, com o intuito de orientar que a via de parto seja escolhida da melhor forma possível, com seus riscos avaliados, para que se consigam os benefícios esperados para cada tipo de parto. 
No Estado do Paraná, a Rede Mãe Paranaense, fundamentada na Rede Cegonha proposta pelo MS em 2011, tem como objetivo captar precocemente as gestantes e fornecer atendimento e acompanhamento adequado para a gestante e a criança especialmente em seu primeiro ano de vida ${ }^{37}$.

Por fim, é importante salientar que muitos são os fatores que podem influenciar a taxa das cesáreas, dificultando a redução dessas taxas a curto prazo. Dentre esses fatores estão o elevado número de cesáreas anteriores, as características individuais (demográficas, clínicas e obstétricas das mulheres), de ordem estrutural ou sistêmica (modelo de atenção obstétrica) e ainda os fatores mais subjetivos (preferências das mulheres e dos profissionais) ${ }^{16}$.

Este estudo apresentou limitações, como a falta da avaliação simultânea de informações não disponíveis no sistema de informações utilizado, como os motivos ou indicações para a realização das cesáreas; complicadores durante a gestação; morbidade e mortalidade materna e neonatal e características socioeconômicas como atendimento pela rede SUS ou sistema de saúde suplementar. Também não se pode extrapolar para outras regiões os resultados encontrados, pelas diferenças existentes entre as populações, dificultando o uso das informações para fins de ações de saúde de uma maneira generalizada.

O trabalho demonstrou que as Regionais de Saúde do Estado do Paraná têm mantido elevadas proporções de cesáreas e grande maioria das regionais apresentou tendência crescente de cesáreas.

Mais avaliações relacionadas ao parto cesáreo devem ser realizadas em populações de diferentes regiões geográficas do país, devido à complexidade e às mudanças que ocorrem com relação às características dos fatores influenciadores na escolha de parto. Além disso, espera-se que os resultados de novas pesquisas incentivem a realização racional e segura das cesáreas, mediante ações interdisciplinares em saúde.

\section{REFERÊNCIAS}

1. World Health Organization. Appropriate technology for birth. Lancet. 1985;2(8452):436-37. PMid:2863457.

2. Betrán AP, Torloni MR, Zhang JJ, Gülmezoglu AM, Aleem HA, Althabe F, et al. WHO statement of caesarean section rates. BJOG. 2016;123(5):667-70. http://dx.doi.org/10.1111/1471-0528.13526. PMid:26681211.

3. Ruiz-Sánchez J, Espino y Sosa S, Vallejos-Parés A, Durán-Arenas L. Cesárea: tendencias y resultados. Perinatol Reprod Hum. 2014 mar;28(1):33-40.

4. Domingues RMSM, Dias MAB, Nakamura-Pereira M, Torres JA, d'Orsi E, Pereira APE, et al. Processo de decisão pelo tipo de parto no Brasil: da preferência inicial das mulheres à via de parto final. Cad Saúde Pública 2014;30(Supl 1):S101-S116. http://dx.doi.org/10.1590/0102-311X00105113.

5. Gibbons LB., Belizán JM, Lauer JA, Betrán AP, Merialdi M, Althabe F. The global numbers and costs of additionally needed and unnecessary cesarean sections performed per year: overuse as a barrier to universal coverage. Geneva:WHO; 2010. (World Health Report. Background Paper; 30).

6. Tian X, Wu J, Li B, Qin M, Qi J. Occurrence of cesarean section and related factors in 40 counties of China from 1978 to 2010. Zhonghua Yu Fang Yi Xue Za Zhi. 2014;48(5):391-5. PMid:24985379.

7. Organização para a Cooperação e Desenvolvimento Econômico. Health at a glance 2011. Paris: OECD Publishing; 2011.

8. Souza JP, Gülmezoglu AM, Lumbiganon P, Laopaiboon M, Carroli G, Fawole B, et al. Caesarean section whithout medical indications is associated with an increased risk of adverse short-term maternal outcomes: the 2004-2008 WHO Global Survey on Maternal and Perinatal Health. BMC Med. 2010;8(1):71. PMid:21067593.

9. Liu S, Liston RM, Joseph KS, Heaman M, Sauve R, Kramer MS. Maternal mortality and severe morbidity associated with low-risk planned cesarean delivery versus planned vaginal delivery at term. Can Med Assoc J. 2007;176(4):455-60. http://dx.doi.org/10.1503/cmaj.060870.

10. Mylonas I, Friese K. Indications for and risks of elective Cesarean Section. Dtsch Arztebl Int. 2015;112(2930):489-95. http://dx.doi.org/10.3238/arztebl.2015.0489. PMid:26249251.

11. Entringer AP, Pinto M, Dias MAB, Gomes MASM. Análise de custo-efetividade do parto vaginal espontâneo e da cesariana eletiva para gestantes de risco habitual no Sistema Único de Saúde. Cad Saude Publica. 2018;34(5):e00022517. PMid:29768579. 
12. Gibbons L, Belizan JM, Lauer JA, Betran AP, Merialdi M, Althabe F. Inequities in the use of cesarean section deliveries in the world. Am J Obstet Gynecol. 2012 abr;206(4):331.e1-19. PMid:22464076.

13. Brasil. Ministério da Saúde. Departamento de Informática do SUS. Sistema de Informação sobre Nascidos Vivos (SINASC) [Internet]. Brasília: DATASUS; 2014 [citado em 2018 set 3]. Disponível em: http://tabnet. datasus.gov.br/cgi/tabcgi.exe?sinasc/cnv/nvuf.def

14. Kleinbaum DG, Kupper LL, Nizan A, Müller KE. Applied regression analysis and other multivariable methods (the Duxbury series in statistics and decision sciences) (2nd ed.). Boston: PWS- Kent, cop.; 1988.

15. Souza JP, Betran AP, Dumont A, Mucio B, Gibbs Pickens CM, Deneux-Tharaux C, et al. A global reference for caesarean section rates (C-Model): a multicountry cross-sectional study. BJOG. 2016 fev;123(3):427-36. http://dx.doi.org/10.1111/1471-0528.13509. PMid:26259689.

16. Brasil. Ministério da Saúde. Secretaria de Ciência, Tecnologia e Insumos Estratégicos. Comissão Nacional de Incorporação de Tecnologias no SUS. Diretrizes de Atenção à Gestante: a operação cesariana [Internet]. Brasília: Ministério da Saúde; 2016 [citado em 2018 set 3]. Disponível em: http://conitec.gov.br/images/ Consultas/Relatorios/2016/Relatorio_Diretrizes_Cesariana_N179.pdf

17. Ye J, Zhang J, Mikolajczyk R, Torloni MR, Gülmezoglu AM, Betrán AP. Association between rates of caesarean section and maternal and neonatal mortality in the 21st century: a worldwide population-based ecological study with longitudinal data. BJOG. 2015;123(5):745-53. http://dx.doi.org/10.1111/1471-0528.13592. PMid:26331389.

18. Villar J, Carroli G, Zavaleta N, Donner A, Wojdyla D, Faundes A, et al. Maternal and neonatal individual risks and benefits associated with caesarean delivery: multicentre prospective study. BMJ. 2007;335(7628):1025. http://dx.doi.org/10.1136/bmj.39363.706956.55. PMid:17977819.

19. Baskett TF, O'Connell CM. Severe obstetric maternal morbidity: a 15-year population-based study. J Obstet Gynaecol. 2005;25(1):7-9. http://dx.doi.org/10.1080/01674820400023408. PMid:16147683.

20. Ramires de Jesus G, Ramires de Jesus N, Peixoto-Filho FM, Lobato G. Caesarean rates in Brazil: what is involved? BJOG. 2015;122(5):606-9. http://dx.doi.org/10.1111/1471-0528.13119. PMid:25327984.

21. Liu Y, Li G, Chen Y, Wang X, Ruan Y, Zou L, et al. A descriptive analysis of the indications for caesarean section in mainland China. BMC Pregnancy Childbirth. 2014;14(1):410-2. http://dx.doi.org/10.1186/ s12884-014-0410-2. PMid:25495550.

22. Zhang J, Troendle J, Reddy UM, Laughon SK, Branch DW, Burkman R, et al. Contemporary cesarean delivery practice in the United States. Am J Obstet Gynecol. 2010;203(4):326.e1-326.e10. http://dx.doi. org/10.1016/j.ajog.2010.06.058. PMid:20708166.

23. Instituto Nacional de Estadísticas e Informática. Perú: encuesta demográfica y de salud familiar [Internet]. Peru: INEl; 2014 [citado em 2018 set 3]. Disponível em: https://www.inei.gob.pe/media/MenuRecursivo/ publicaciones_digitales/Est/Lib1211/pdf/Libro.pdf

24. Althabe F, Sosa C, Belizán JM, Gibbons G, Jacquerioz F, Bergel E. Cesarean section rates and maternal and neonatal mortality in low-, medium-, and high-income countries: an ecological study. Birth. 2006;33(4):2707. http://dx.doi.org/10.1111/j.1523-536X.2006.00118.x. PMid:17150064.

25. Teixeira A, Machado HS. WHO caesarean section rate: relevance and ubiquity at the present day. J Pregnancy Child Health. 2016;3(2):233. http://dx.doi.org/10.4172/2376-127X.1000233.

26. Gagnon AJ, Van Hulst A, Merry L, George A, Saucier JF, Stanger E, et al. Cesarean section rate differences by migration indicators. Arch Gynecol Obstet. 2013;287(4):633-9. http://dx.doi.org/10.1007/s00404-0122609-7. PMid:23132050.

27. Rattner D, Moura EC. Nascimentos no Brasil: associação do tipo de parto com variáveis temporais e sociodemográficas. Rev Bras Saúde Mater Infant. 2016;16(1):39-47. http://dx.doi.org/10.1590/180693042016000100005.

28. Madeiro A, Rufino AC, Santos AO, Madeiro A, Rufino AC, Santos AO. Cesarean sections in Piauí State: trend and associated factors in the period 2000-2011, Brazil. Epidemiol Serv Saude. 2017;26(1):81-90. http:// dx.doi.org/10.5123/S1679-49742017000100009. PMid:28226010.

29. Barros FC, Matijasevich A, Maranhão AG, Escalante JJ, Rabello DL No, Fernandes RM, et al. Cesarean sections in Brazil: will they ever stop increasing? Rev Panam Salud Publica. 2015;38(3):217-25. PMid:26758000.

30. Silva ALA, Mendes ACG, Miranda GMD, Santos PM No. Assistência ao parto no Brasil: uma situação crítica ainda não superada. 1999-2013. Rev Bras Saúde Mater Infant. 2016;16(2):139-48.

31. Hellerstein S, Feldman S, Duan T. China's 50\% caesarean delivery rate: is it too high? BJOG. 2015;122(2):1604. http://dx.doi.org/10.1111/1471-0528.12971. PMid:25138909. 
32. Soto-Vega E, Urrutia-Osorio M, Arellano-Valdez F, López BIY, Hernández RCH. The epidemic of the cesarean section in private hospital in Puebla, México. Obstet Gynecol Int J. 2015;2(6):1-5. http://dx.doi. org/10.15406/ogij.2015.02.00058.

33. Shabila NP. Rates and trends in cesarean sections between 2008 and 2012 in Iraq. BMC Pregnancy Childbirth. 2017;17(1):22. PMid:28077096.

34. World Health Organization. WHO statement of caesarean section rates [Internet]. Geneva: WHO; 2015 [citado em 2018 set 3]. Disponível em: http://apps.who.int/iris/bitstream/handle/10665/161442/WHO_ RHR_15.02_eng.pdf?sequence $=1$

35. World Health Organization. Guia de Implementação da Lista de Verificação da OMS para Partos Seguros: melhorar a qualidade dos partos realizados em unidades de saúde para as mães e os recém-nascidos [Internet]. Geneva: WHO; 2017 [citado em 2018 set 3]. Disponível em: http://apps.who.int/iris/bitstre am/10665/199177/5/9789248549458-por.pdf

36. Brasil. Agência Nacional de Saúde Suplementar. Cartilha nova organização do cuidado ao parto e nascimento para melhores resultados de saúde: Projeto Parto Adequado- fase 1 [Internet]. Rio de Janeiro: ANS; 2016 [citado em 2018 set 3]. Disponível em: http://www.ans.gov.br/images/stories/Materiais_para_ pesquisa/Materiais_por_assunto/web_total_parto_adequado.pdf

37. Paraná. Secretaria de Estado da Saúde do Paraná. Programa Rede Mãe Paranaense: Linha guia [Internet] Paraná: SESA; 2012 [citado em 2018 set 3]. Disponível em: http://www.saude.pr.gov.br/ arquivos/File/ MaeParanaense_2014_LinhaGuia_Ed03_148x210mm_1.pdf 